\section{Cryopreservation: A Strategy Technique for Safe Preservation of Genetically Transformed Plant Materials}

\section{Qiaochun Wang ${ }^{1 *}$ Renrui Wang ${ }^{1}$, Baiquan $\mathrm{Li}^{1}$ and Zhenhua Cui $^{1}$}

'State Key Laboratory of Crop Stress Biology for Arid Region, College of Horticulture, Northwest A\&F University, China

The world population now is 6.7 billion and is predicted to reach 9 billion by 2050 [1]. Such a rapid growing population has tremendously increased the challenge for food security. Obviously, it is impossible for traditional agriculture to ensure the food security, while plant biotechnology offers considerable potential to realize this goal [2]. Commercial cultivation of genetically modified (GM) crops started in 1996. Since then, cultivation of GM crops has rapidly increased and the total cultivation areas of GM crops had reached 134 million ha by 2009 [3]. Over the last 15 years, great benefits have been brought to sustainable agriculture by commercial cultivation of genetically modified (GM) crops [2]. Further development of new GM crops will with no doubt contribute to meeting the requirements for food by the increasing population [3]. In vitro plant cell cultures have been for a long time used for producing natural products including proteins [4]. Expression of foreign gene (s) in plant cell cultures by genetic transformation, which enables production of recombinant proteins, i.e. molecular farming [5], opened a new avenue for production of therapeutically valuable proteins and over the past three decades great progresses have been made in the said subject [4]. To date, molecular farming products are already commercially available or in more advanced clinical stage [6].

Cryopreservation refers to storage of living cells, tissues or organs at ultra-low temperatures, usually that of liquid nitrogen $\left(-196^{\circ} \mathrm{C}\right)$ [4]. When stored at such a low temperature, cellular divisions and metabolic processes of the living cells arrest and therefore, plant materials can be conserved for an indefinite period of time, while maximally maintaining their genetic stability [7]. Furthermore, cryopreservation occupies little space, eliminates contamination and demands only maintenance. Therefore, cryopreservation has been considered an ideal means for safe and long-term storage of plant germplasm $[7,8]$.

Safe maintenance of transgenic materials has been a serious problem, especially in developing countries including China. Transgenic materials are frequently maintained either in vitro or in vivo before being analyzed, evaluated and finally released for

\footnotetext{
*Corresponding author: Qiaochun Wang, State Key Laboratory of Crop Stress Biology for Arid Region, College of Horticulture, Northwest A\&F University, Yangling 712100, Shaanxi, China, Tel: +86-29-87081660; Fax: +86-2987081660; E-mail: qiaochunwang@nwsuaf.edu.cn
}

Received: July 16, 2012 Accepted: July 18, 2012 Published: July 25, 2012 commercial production. Maintenance of transgenic materials by repeated subculture is time-consuming and labor-cost, and may cause culture loss due to contamination or human error. More importantly, in vitro or in vivo maintenance may create an extra risk of transgene loss or gene flow [9]. Therefore, establishment of safe and long-term preservation technique of transgenic materials, which is capable of ensuring genetic integrity of transgenes and avoiding gene flow, is of significant importance. The first study attempting to cryopreserve transgenic rice (Oryza sativa) with NPTII and GUS gens was reported by Meijer et al. [10]. Since then, there have been a number of studies on cryopreservation of transgenic materials containing different target genes and including field crops like Oryza sativa and Triticum aestivum, fruit crops such as Carica papaya, Citrus sinensis and Pyrus pyrifolia, ornamental crops like Betula pendula, and medicinal crops like Papaver somniferum [9]. Successful cryopreservations have been reported for transgenic cell suspensions that expressed human serum albumin (HAS) in Nicotiana tabacum [11], Escherichia coli heat labile enterotoxin (LT) protein in N. tabacum [12] and hCTlA4Ig in O. sativa [13]. All results obtained so far all indicate that cryopreservation does not affect expression of foreign genes in transgenic materials and the productive ability of cryopreserved cells containing recombinant proteins was similar to that in non-cryopreserved cultures [11-13], thus allowing transgenic materials to be stored in a safe manner before being analyzed and evaluated and establishment of stable seed stocks for commercial production of homologous proteins [9]. Results obtained so far all indicate that cryopreservation does not affect expression of foreign genes and allows transgenic materials to be stored in a safe manner before being analyzed and evaluated [6].

With a motive of preservation of plant germplasm, the first report on successful cryopreservation of plants was published in 1960 [14]. Since then, various cryopreservation techniques such as droplet, droplet-vitrification, vitrification, encapsulation-dehydration and encapsulation-vitrification have been successfully developed for almost all economically important crops including woody and herbaceous plants ranging from tropical to temperate regions [7]. The main limitation involved in cryopreservation techniques is a strong genotypic response. Nevertheless, wide application of cryotechniques to transgenic materials helps ensure their maintenance [9]

\section{References}

1. von Braun J (2010) Food insecurity, hunger and malnutrition: necessary policy and technology changes. N Biotechnol 27: 449-452.

2. Fedoroff NV (2010) The past, present and future of crop genetic modification. N Biotechnol 27: 461-465.

3. James C (2010) A global overview of biotech (GM) crops: adoption, impact and future prospects. GM Crops 1: 8-12.

4. Xu JF, Ge XM, Dolan MC (2011) Towards high-yield production of pharmaceutical protein with plant cell suspension cultures. Biotechnol Adv 29: 278-299.

5. Franken E, Teuschel U, Hain R (1997) Recombinant proteins from transgenic plants. Curr Opin Biotechnol 8: 411-416.

6. Spök A, Karnar S (2008) Plant molecular farming: Opportunities and challenges. JRC Scientific and Technical Reports.

7. Benson E (2008) Cryopreservation of phytodiversity: a critical appraisal of 
Citation: Wang Q (2012) Cryopreservation: A Strategy Technique for Safe Preservation of Genetically Transformed Plant Materials. Adv Genet Eng Biotechnol 1:1.

theory \& practice. Crit Rev Plant Sci 27: 141-219.

8. Reed BM (2008) Plant Cryopreservation: A Practical Guide, Springer, New York.

9. Wang B, Zhang Z, Yin Z, Feng C, Wang Q (2012) Novel and potentia application of cryopreservation to plant genetic transformation. Biotechnol Adv 30: 604-612.

10. Meijer EGM, Iren E, Schrijnemakers E, Hensgens LAM, van Zijderveld M, et al. (1991) Retention of the capacity to produce plants from protoplasts in cryopreserved cell lines of rice (Oryza sativa L). Plant Cell Rep 10:171-174.

11. Schmale K, Rademacher TH, Fischer R, Hellwig S (2006) Towards industrial usefulness - Cryocell banking of transgenic BY-2 cell cultures. J Biotechnol 124: 302-311.

12. Van Eck J, Keen $P$ (2009) Continued expression of plant-made vaccines following long-term cryopreservation of antigen-expressing tobacco cell cultures. In Vitro Cell Dev Biol-Plant 45: 750-757.

13. Cho JS, Hong SM, Joo SY, Yoo JS, Kim DI (2007) Cryopreservation of transgenic rice suspension cells producing recombinant $h C T L A 4 / g$. Appl Microbiol Biotechnol 73: 1470-1476

14. Sakai $A$ (1960) Survival of the twigs of woody plants at $-196{ }^{\circ} \mathrm{C}$. Nature 185 392-394.

\section{Author Affiliations}

${ }^{1}$ Department of Structural Biology, University of Vienna, Vienna, Austria ${ }^{2}$ Department of Neurology, University of Colorado, Anschutz Medical Campus, Aurora, USA

${ }^{3}$ Key Laboratory of Major Diseases in Children and National Key Discipline of Pediatrics, Ministry of Education, Beijing Pediatric Research Institute, Beijing Children's Hospital, Capital Medical University, Beijing, China ${ }^{4}$ Division of Hygiene and Medical Microbiology, Department of Hygiene,

Microbiology and Social Medicine, Innsbruck, Austria

Submit your next manuscript and get advantages of SciTechnol submissions

* 50 Journal

* 21 Day rapid review process

* 1000 Editorial team

* 2 Million readers

* More than 5000 lactbats

- Publication immediately after acceptance

* Quality and quick editorial, review processing

Submit your next manuscript at • www.scitechnol.com/submission 